\title{
Stochastic Numerical Investigations for a Food Web System of Prey-Predation, Competition and Commensalism
}

\section{Zulqurnain Sabir}

Hazara University

Seshagiri Rao N ( $\sim$ seshu.namana@gmail.com )

Adama Science and Technology University https://orcid.org/0000-0003-2409-6513

Kalyani K

Vignan's Foundation for Science Technology and Research

\section{Research Article}

Keywords: Three species food web system, Nonlinear di囚erential system, Numerical computing, Neural networks, Levenberg-Marquardt backpropagation.

Posted Date: December 6th, 2021

DOI: https://doi.org/10.21203/rs.3.rs-683771/v1

License: (c) (i) This work is licensed under a Creative Commons Attribution 4.0 International License. Read Full License 


\title{
Stochastic numerical investigations for a food web system of prey-predation, competition and commensalism
}

\author{
Zulqurnain Sabir ${ }^{1^{*}}$, N. Seshagiri Rao ${ }^{2^{*}}$ \& K. Kalyani ${ }^{3}$ \\ ${ }^{1}$ Department of Mathematics and Statistics \\ Hazara University, Mansehra, Pakistan. \\ ${ }^{2}$ Department of Applied Mathematics \\ School of Applied Natural Sciences \\ Adama Science and Technology University \\ Post Box No.1888, Adama, Ethiopia. \\ ${ }^{3}$ Department of Mathematics \\ Vignan's Foundation for Science, Technology \& Research \\ Vadlamudi-522213, Andhra Pradesh, India. \\ E-mail: 'zulqurnain_maths@hu.edu.pk, ${ }^{2}$ seshu.namana@gmail.com \\ \& 33alyani.namana@gmail.com
}

\begin{abstract}
Employing Levenberg-Marquardt backpropagation(LMB) neural network, the system of three species nonlinear equations are illuminated by designing an integrated numerical computing-based plot. The proposed dynamical system comprises of two competing species which are growing logistically in nature and, the third species is predating with Holling type II functional response mode on second species and also acts host for the first prey species. Besides, the prey species protect themselves to refuge high predation. The designed LMB neural network has been used to exhibits the solutions of the dynamical frame work. In each case of the species, a reference dataset of the planned LMB neural network is initiated in comparison of Adam numerical program. The approximate results of the food web system are displayed within the training, confirmation and testing strategies to redesign the neural network to minimize the mean square error (MSE) function employing the designed LMB. The investigations depend on the corresponding achievements and the examinations based on MSE out comes, correlation, regression and error histograms signify the proficiency, rightness as well as the potency of the suggested LMB neural network conspire.
\end{abstract}

Keywords: Three species food web system; Nonlinear differential system; Numerical computing; Neural networks; Levenberg-Marquardt backpropagation.

\section{Introduction}

In recent years, the study of environmental models has achieved huge significance between the researchers. The two species models are considered due to the one sort of relationship 
Table 1: The biological interpretation of parameters and variables.

\begin{tabular}{|l|l|}
\hline Parameters and variables & Interpretation \\
\hline $\mathscr{X}, \mathscr{Y}$ & The densities of logistically growing competing species. \\
\hline $\mathscr{Z}$ & The density of a third species which is predating on $\mathscr{Y}$. \\
\hline$r_{i}(i=1,2)$ & $\mathscr{X}$ and $\mathscr{Y}$ intrinsic growth rates. \\
\hline $\mathscr{K}_{i}(i=1,2)$ & $\mathscr{X}$ and $\mathscr{Y}$ species carrying capacities. \\
\hline$p$ & The refuge proportion parameter from predation. \\
\hline$a$ & Attack rate of the single predator $(\mathscr{X})$ search for single prey $(\mathscr{X})$. \\
\hline$b$ & Half saturation level of $\mathscr{\mathscr { X }}$ over $\mathscr{X}$. \\
\hline$\alpha_{i j}(i, j=1,2 ; i \neq j)$ & Competitive coefficients of $\mathscr{X}$ and $\mathscr{Y}$. \\
\hline$\delta_{13}$ & The coefficient of commensal. \\
\hline
\end{tabular}

by the time as prey-predation, competition, mutualism, commensalism etc. In particular, the prey-predator models of different Holling types are investigated in [1-5]. Also various functional responses of Leslie-Gower, Beddington-DeAngelis and ratio-dependent are broadly examined in the references [6-12]. The prey species protect themselves from the unrestricted risk of predation by refusing. Normally "refuge" can be any strategy/procedure which is used to reduce the predation risk. Various authors have considered in this direction of refuge of the prey species in their models [13-15]. The investigation work of two species interactions of mutualism, competition and commensalism can be seen from the references [16-22], which explored the behavior of nonlinear systems under the environmental conditions by studying analytically/numerically.

Later, the above said interactions are extended for food chain/web systems of three species, the readers may refer to [23-32]. In addition, multi-interaction of food-web frameworks involving three species create much interest as compared with the systems of two species in terms of perceive the quasi-periodic solutions, strange attractors and chaos [33-35]. A few food chains considering mutualism interaction of the species are investigated in $[36,37]$ and explored the existence of the species analytically and also numerically in nature. Furthermore, the sustainability of three species food chain of preypredation and commensalism under environmental conditions can be found from [38-40]. Some authors in $[26,28,33,41]$ have considered more than one species refuge to protect from the predation in three species food chain models for further investigations.

A food chain frame work comprises of competition, commensalism and prey-predation is basically a nonlinear model which is given in [42] as below:

$$
\begin{aligned}
& \frac{d \mathscr{X}}{d \tau}=r_{1} \mathscr{X}\left[1-\frac{\mathscr{X}}{\mathscr{K}_{1}}-\frac{\alpha_{12} \mathscr{Y}}{\mathscr{K}_{1}}\right]+\delta_{13} \mathscr{X} \mathscr{X}, \\
& \frac{d \mathscr{Y}}{d \tau}=r_{2} \mathscr{Y}\left[1-\frac{\mathscr{Y}}{\mathscr{K}_{2}}-\frac{\alpha_{21} \mathscr{Y}}{\mathscr{K}_{2}}\right]-\frac{a(1-p) \mathscr{Y} \mathscr{X}}{b+(1-p) \mathscr{Y}}, \\
& \frac{d \mathscr{E}}{d \tau}=\mathscr{E}\left[-e+\frac{a c(1-p) \mathscr{Y} \mathscr{\mathscr { X }}}{b+(1-p) \mathscr{Y}}\right] .
\end{aligned}
$$

with the positive initial conditions: $\mathscr{X}(0) \geq 0, \mathscr{Y}(0) \geq 0, \mathscr{Z}(0) \geq 0$. The parameters and the variables of the above food web (1) are given in Table 1. The non-dimensionalized equations of (1) are

$$
\begin{aligned}
& \frac{d x}{d t}=x\left[1-x-\beta_{12} y\right]+\delta x z=x \psi_{1}(x, y, z)=\mathcal{P}_{1}(x, y, z), \\
& \frac{d y}{d t}=r y\left[1-y-\beta_{21} x\right]-\frac{(1-p) y z}{\mathrm{w}_{1}+(1-p) y}=y \psi_{2}(x, y, z)=\mathcal{P}_{2}(x, y, z), \\
& \frac{d z}{d t}=z\left[-\mathrm{w}_{2}+\frac{\mathrm{w}_{3}(1-p) y z}{\mathrm{w}_{1}+(1-p) y}\right]=z \psi_{3}(x, y, z)=\mathcal{P}_{3}(x, y, z),
\end{aligned}
$$


and the related positive conditions are: $x(0) \geq 0, y(0) \geq 0, z(0) \geq 0$ with respect to the below non-dimensional parameters, variables;

$$
\begin{aligned}
& t=r_{1} \tau, x=\frac{\mathscr{X}}{\mathscr{K}_{1}}, y=\frac{\mathscr{Y}}{\mathscr{K}_{2}}, z=\frac{a \mathscr{E}}{r_{1} \mathscr{K}_{2}}, \\
& \mathrm{w}_{1}=\frac{b}{\mathscr{K}_{2}}, \mathrm{w}_{2}=\frac{e}{r_{1}}, \mathrm{w}_{3}=\frac{a c}{r_{1}}, r=\frac{r_{1}}{r_{2}}, \\
& \beta_{12}=\frac{\alpha_{12} \mathscr{K}_{2}}{\mathscr{K}_{1}}, \beta_{21}=\frac{\alpha_{21} \mathscr{K}_{1}}{\mathscr{K}_{2}}, \delta=\frac{\delta_{13} \mathscr{K}_{2}}{a} .
\end{aligned}
$$

The food web framework has been illuminated analytically/numerically by utilizing diverse schmes but stochastic LMB neural network approach has never been applied to solve this model. Stochastic approaches are effective to illuminate numerous complex models utilizing the swarming/evolutionary approaches such as singular higher order models [4345], dusty plasma models [46], biological models [47-50], fluid dynamic problems [51,52], electric circuits [53], singular three-point model [54] and periodic differential model [55]. The potential visualizations of the proposed LMB neural network are given as:

- A novel integrated design of an intelligent computing conspire is bestowed by means of modeling competency of LMB neural network connected to scrutinize the dynamics of three species framework represented with set of nonlinear ordinary differential equations.

- The outlined LMB neural networks work successfully on a information set created from numerical Adam method for diverse variations of the nonlinear modeling framework.

- The execution by means of comparative examinations from reference results of Adam strategy on mean square error (MSE), regression, error histograms and correlation metrics set up the worth of outlined LMB neural networks.

- Advantage of the proposed LMB neural network strategy is the smooth execution, straight forwardness of the concept, stability and exhaustive appropriateness.

The remaining paper is categorized as follows: Sect. 2 shows the solution of LMB neural network approach is implemented to illuminate the dynamical framework. Sect. 3 represents the planned LMB neural network with basic clarifications. Finally, Sect. 4, the concluding explanations with inactive associated studies beside the scope of future research work are expressed

\section{Methodology}

The methodology based on the procedures of LMB neural network is provided in two steps as:

- The necessary interpretations are given to construct or develop the LMB neural systems dataset by the utilized of standard numerical strategies i.e., Runge-Kutta or Adam numerical solvers.

- Implementation method endorsed for LMB neural networks is presented to discover the approximate solution of the model exhibited in the set of Equations (2).

The plan of workflow of the LMB neural network approach is plotted in Figure 1, which could be a blend of multi layer development of neural systems under the optimization of LMB strategy. A framework for one neuron based on the neural network methodology is accessible in Figure 2 in the of input, output and hidden layers structure. The proposed LMB neural networks are implemented utilizing the "nftool" schedule within the "Matlab" package for the proper sets of validation data, hidden neurons, testing data and learning schemes. 


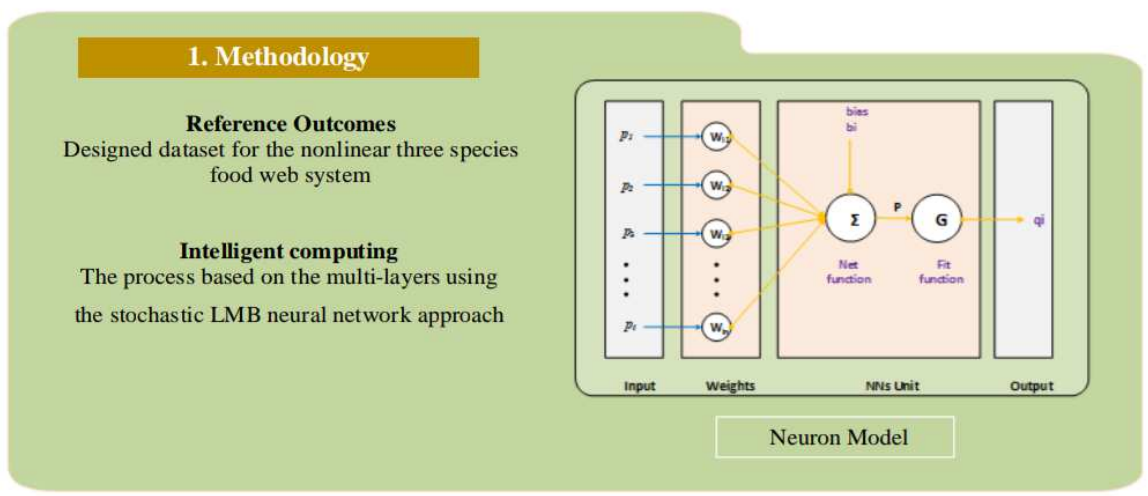

\section{Results Simulations}
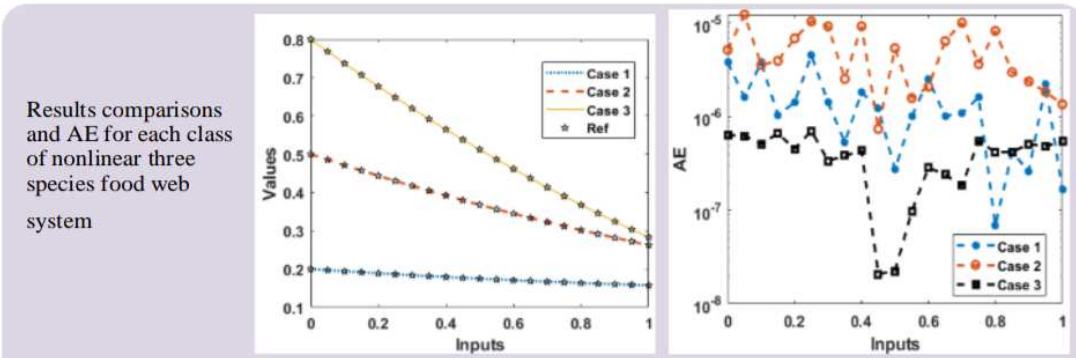

Result Comparison

$\mathrm{AE}$

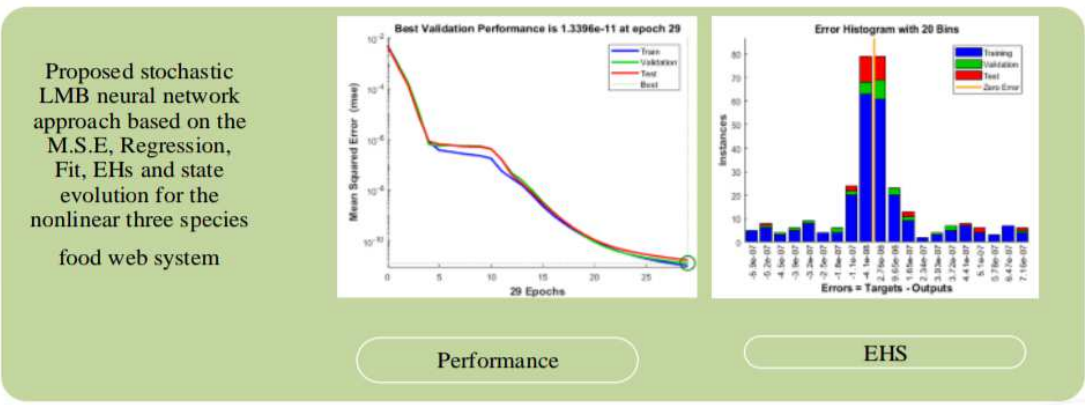

Figure 1: schematic chart of the LMB proposed neural network for all three species $x, y$ and $z$ of the dynamical system (2).

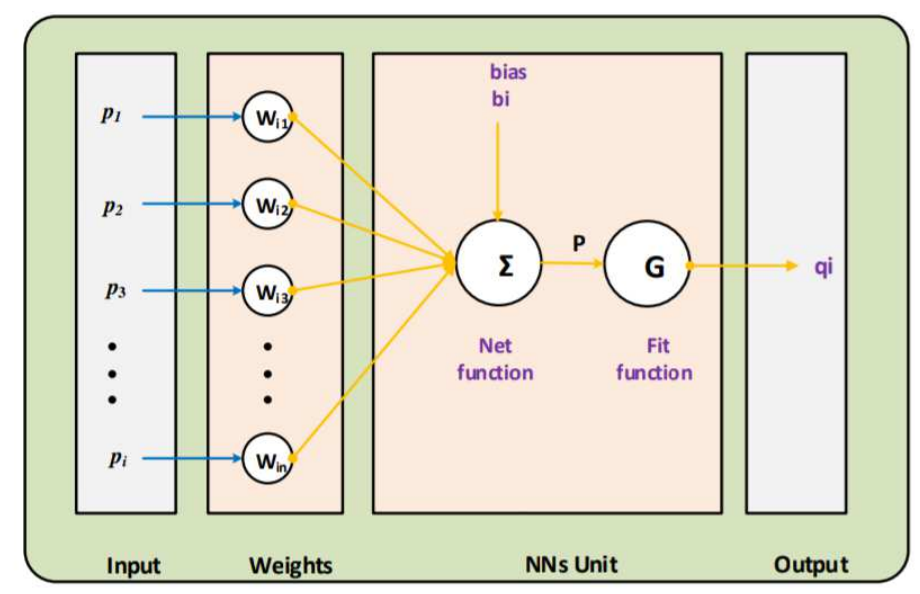

Figure 2: Outlined show framework based on the single neuron. 


\section{Numerical Measures with Analysis}

In this section, we illuminate the food web framework numerically utilizing the LMB neural network. Three distinctive cases based up on the initial conditions of system (2) are taken as

$$
\begin{aligned}
& \text { Case 1: } x(0)=0.1 ; y(0)=0.2 ; z(0)=0.3, \\
& \text { Case 2: } x(0)=0.4 ; y(0)=0 . ; z(0)=0.6, \\
& \text { Case 3: } x(0)=0.7 ; y(0)=0.8 ; z(0)=0.9,
\end{aligned}
$$

while the rest of the values that have been used to solve the model are $\beta_{12}=0.4, \delta=0.04$, $r=0.5, \beta_{21}=0.1, \mathrm{w}_{1}=0.14, p=0.4, \mathrm{w}_{2}=0.32009$ and $\mathrm{w}_{3}=0.5$. The proposed numerical result has been acquired employing the LMB neural network of 0.01 step size in the interval [1] for the nonlinear framework. The LMB outlined neural network approach is competent of fathoming the framework given in (2) utilizing "nftool" with 9 coveredup neurons, $70 \%$ values of data training, $15 \%$ testing and $15 \%$ acceptance of the LMB optimization handle. The LMB designed neural network is displayed in Figure 3, and the LMB neural network approach was executed to illuminate each case of the system.

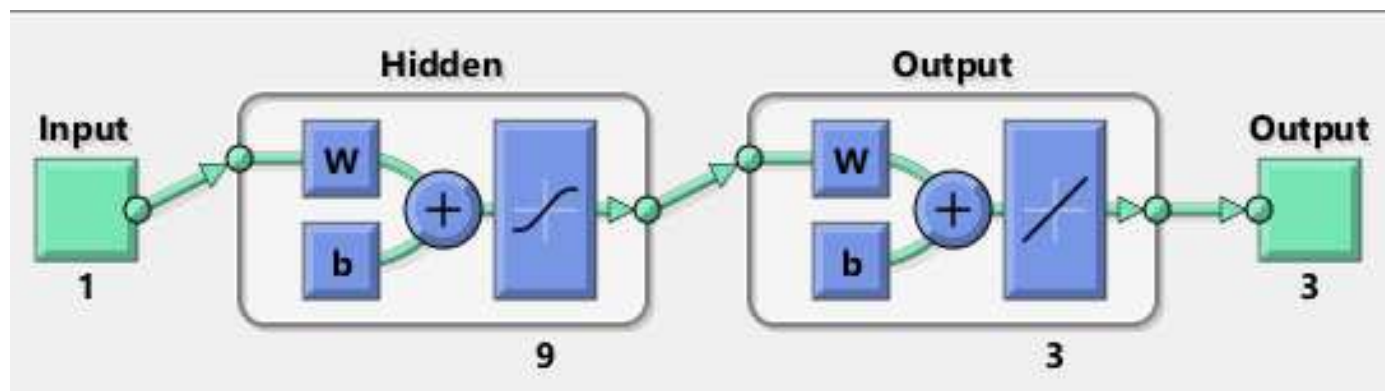

Figure 3: The construction of LMB designed neural network for the food web system.

The LMB outlined neural network for the nonlinear system (2) is plotted in Figures 4-12. The results of the model utilizing the execution and transition states are given in Figures $4 \& 5$. The values of MSE training, testing, best curve and validation are displayed for each case of the model in Figure 4.

The network's best performances at epochs 29, 58, 49 lie around $10^{-11}, 10^{-11}, 10^{-14}$ individually. The gradient-based values utilizing the step measure $(\mathrm{Mu})$ of the designed LMB process given for the framework are $\left[9.90 \times 10^{-08}, 9.82 \times 10^{-08}\right.$ and $9.64 \times$ $\left.10^{-08}\right]$ and $\left[10^{-11}, 10^{-10}, 10^{-13}\right]$ and are appeared in Figure 5. These plots assign the specificity as well as the convergence of the LMB neural network for all cases of the framework.

Figures 6 to 8 approve the fitting plots for each species that appears the result comparisons acquired by the LMB neural network and the numerical Adam results for all the cases of the system. The most extreme values of the error utilizing training, testing and validation inputs of the LMB neural network lie between $10^{-05}$ to $10^{-07}$ being each species of the framework. Furthermore, Figure 9 exhibits the error histograms (EHs) values of the species.

The regression values can be noticed in Figure 10 for each case of the framework. The correlation examinations were applied to make the regression analysis study. It was seen that the correlation values of " $\mathrm{R}$ " lie near to 1 , appearing the perfect form of the modeling, validation, testing and training. The regression examination generally appeared the correctness of the LMB neural network in order to illuminate the dynamical framework.

Additionally, convergence values in MSE sense were accomplished for training, validation, testing, performances, backpropagation, complexity and executed epochs arranged in Table 2 for the dynamical framework. 
Table 2: SNN-LMB neural network results for all cases of the framework (2).

\begin{tabular}{|c|c|c|c|c|c|c|c|c|}
\hline \multirow{2}{*}{ Case } & \multicolumn{3}{|c|}{ MSE } & \multirow{2}{*}{ Performance } & Gradient & Mu & Epoch & Time \\
\cline { 2 - 9 } & Training & Validation & Testing & & & \\
\hline 1 & $1.03 \times 10^{-11}$ & $1.33 \times 10^{-11}$ & $1.77 \times 10^{-11}$ & $1.03 \times 10^{-11}$ & $9.90 \times 10^{-08}$ & $1.00 \times 10^{-11}$ & 29 & 1 \\
2 & $2.20 \times 10^{-11}$ & $7.54 \times 10^{-11}$ & $3.63 \times 10^{-11}$ & $2.21 \times 10^{-12}$ & $9.82 \times 10^{-08}$ & $1.00 \times 10^{-10}$ & 58 & 2 \\
3 & $6.75 \times 10^{-14}$ & $5.79 \times 10^{-14}$ & $5.30 \times 10^{-14}$ & $6.76 \times 10^{-14}$ & $9.64 \times 10^{-08}$ & $1.00 \times 10^{-13}$ & 49 & 3 \\
\hline
\end{tabular}

The comparative results of all three species of the system with respect to Adam strategy and LMB neural network are plotted in Figure 11. The consequences of each case of the dynamical framework are given in Figure 11.

The plots of $x(t), y(t)$ and $z(t)$ within the framework (2) are given in Figures 11(a)(c). It was noticed that the obtained form of the results overlapped with the Adam results for each case of the framework (2). This complete overlapping of the results assigns the flawlessness and correctness of the proposed approach. The absolute error (AE) plots of all three species of the model in all distinct cases can be seen in Figure 12. Within the dynamical framework, the AE values for the parameters $x(t), y(t)$ and $z(t)$ lie thought $\left[10^{-05}, 10^{-08}\right]$. These results demonstrate the exactness of the LMB designed neural network conspire.
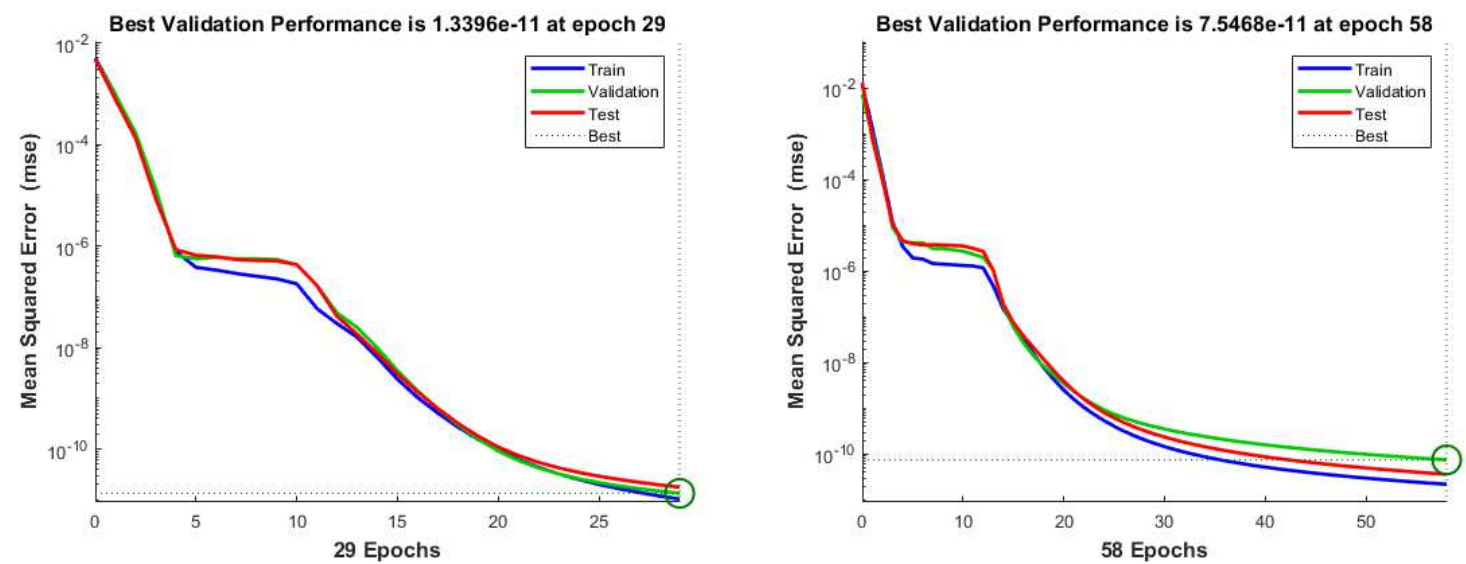

(a) MSE results of the system (2) for case 1. (b) MSE results of the system (2) for case 2.

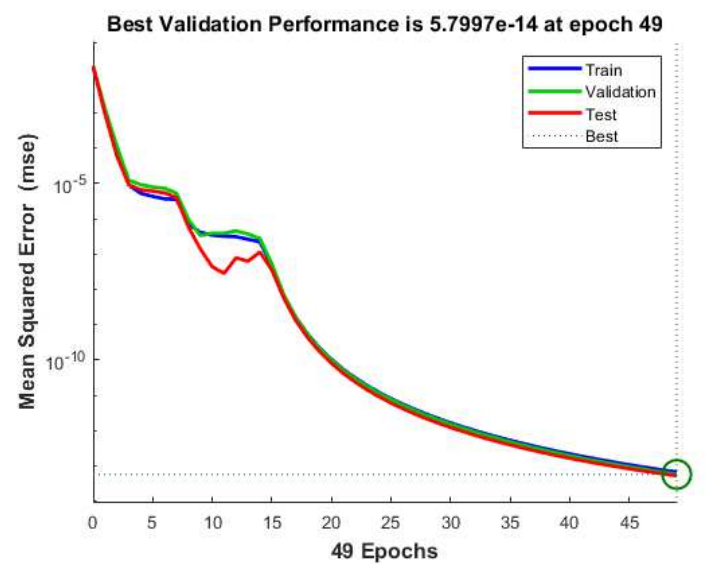

(c) MSE results of the system (2) for case 3 .

Figure 4: Performance curves of the food web system (2) with respect to MSE utilizing the designed LMB neural networks. 

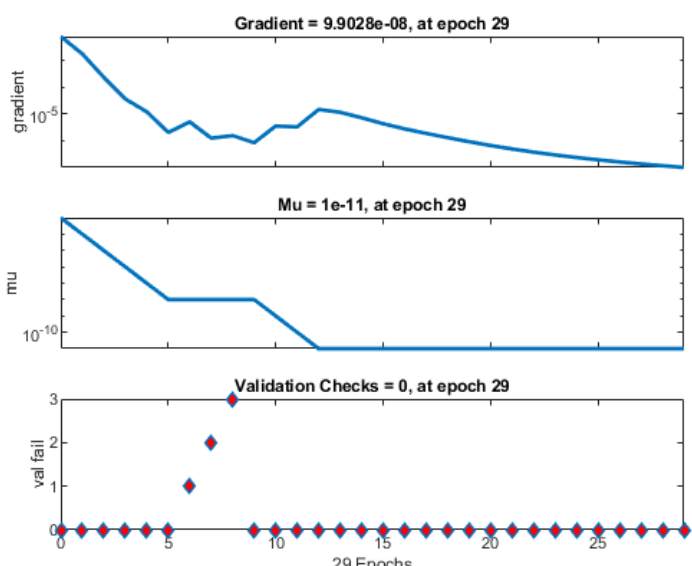

29 Epochs

(a) State transition for case 1 of the system (2). (b) State transition of the system (2) at case 2.
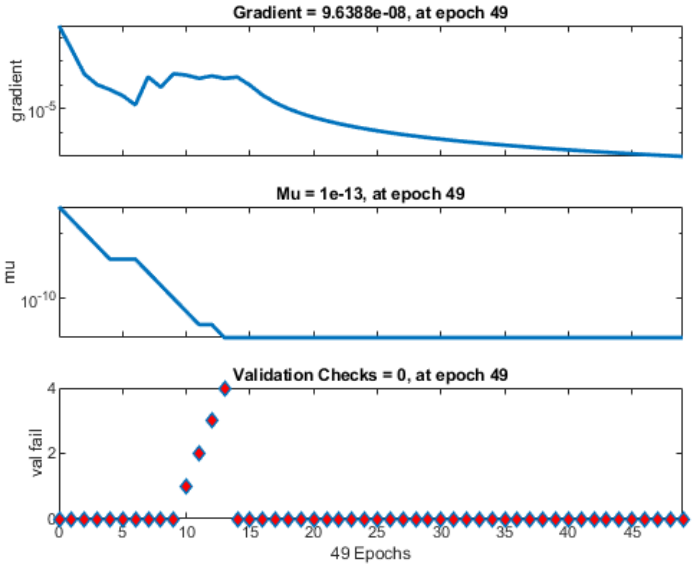

(c) State transition of the system (2) for case 3.

Figure 5: All species state transition of the designed LMB neural networks.

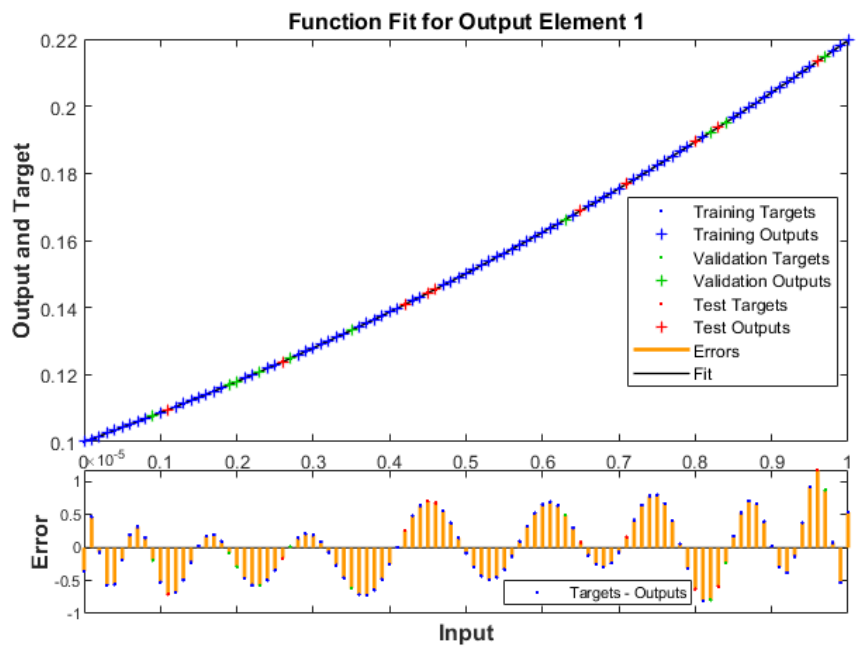

Figure 6: Comparison between LMB neural network and the exact solutions of the dynamical frame work (2) at case 1.

\subsection{Conclusions}

In this work, the food web system of nonlinear conditions were solved by utilizing the combinations of the integrated intelligent computing numerical LMB approach and neu- 


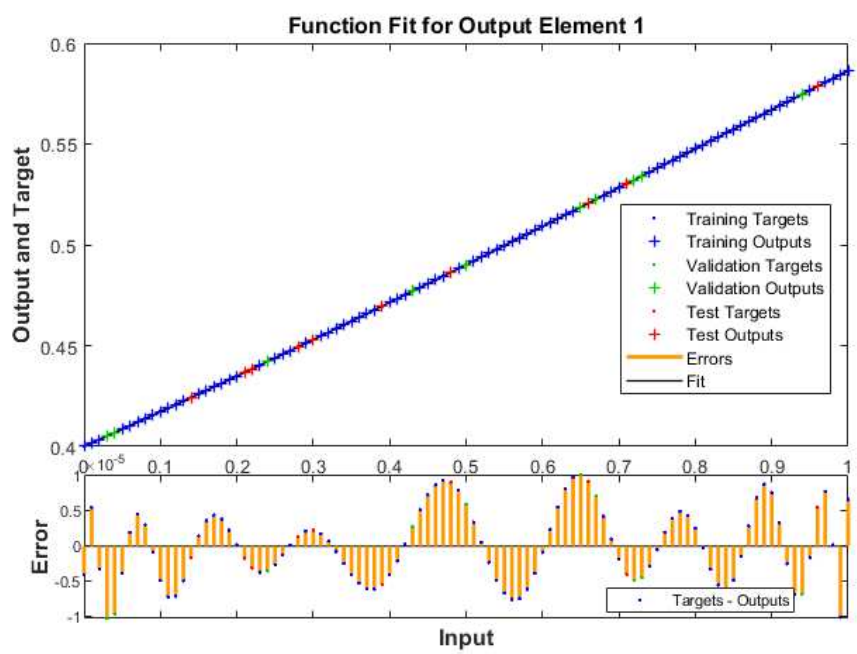

Figure 7: Comparison between LMB neural network and the exact solutions of the dynamical frame work (2) at case 2 .

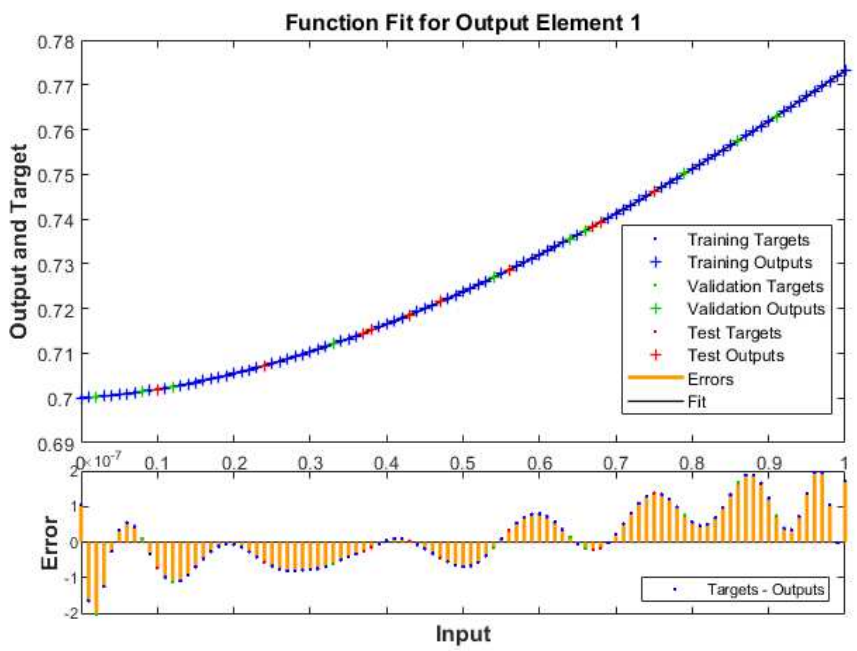

Figure 8: Comparison between LMB neural network and the exact solutions of the dynamical frame work (2) at case 3 .

ral networks. The subjective information of $70 \%$ were utilized for training, whereas the $15 \%$ and $15 \%$ of validation and testing information were utilized, separately, to alter the designed LMB neural network with 9 hidden neurons. In order to evaluate the flawlessness and correctness, the overlapping of the procured results through the designed LMB neural network address with the Adam reference results was drawn. To evaluate the performance through convergence, the values based on mean square error were utilized for the testing, training, best curve and validation for all cases of the framework. The performance of correlation is given to observe the regression examination. The values of the gradient together with the step size were performed for all cases of the framework. Besides, the exactness and precision were certified utilizing the numerical as well as graphical setups of convergence plots, regression dynamics on the error histograms and MSE index, respectively.

In future, the design of LMB neural network can be applied to solve the fluid dynamical [56-60] and fractional systems [61-64]. 


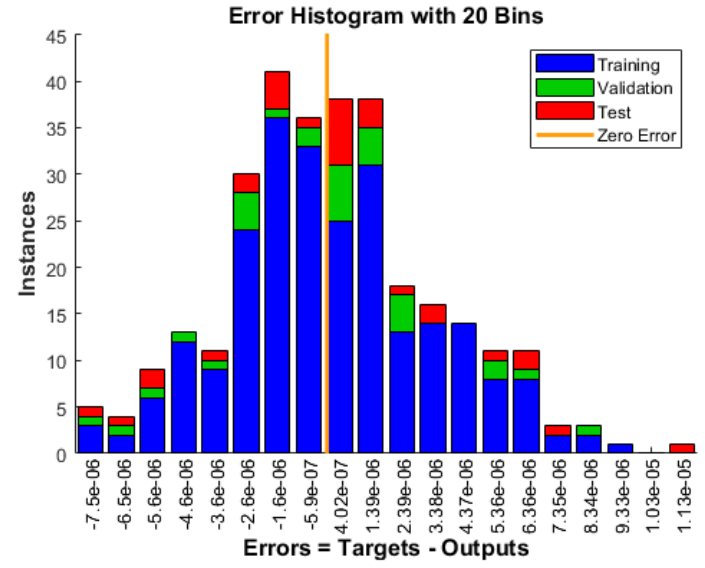

(a) EHs for case 1 of the system (2).

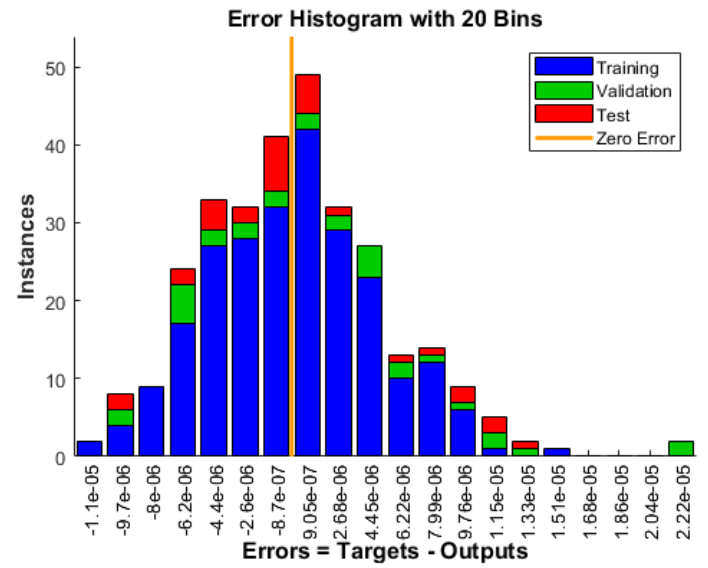

(b) EHs for case 2 of the system (2).

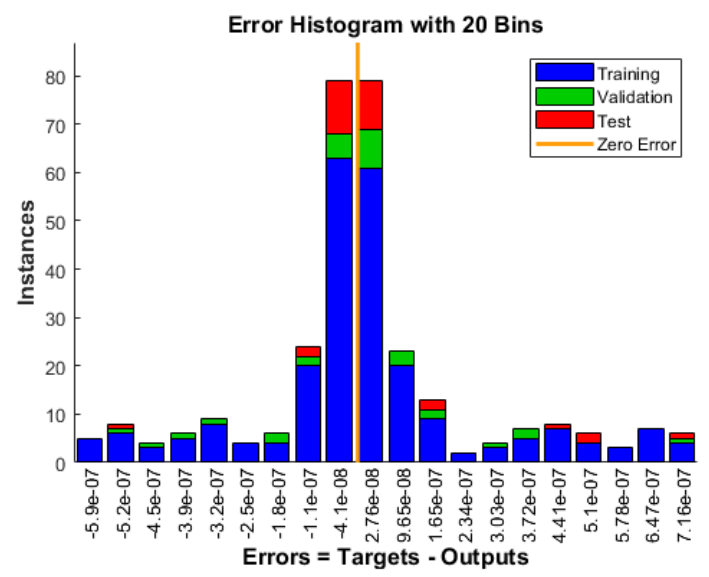

(c) EHs for case 3 of the system (2).

Figure 9: The error histograms (EHs) plots of the food web system (2) with respect to the designed LMB neural networks in each case.

\section{Compliance with Ethical standards}

\section{Abbreviations}

Not applicable.

\section{Funding}

Not applicable

\section{Availability of data and materials}

Not applicable.

\section{Discloser of Potential conflicts of interest}

None (Authors' have no complicit of interest) 

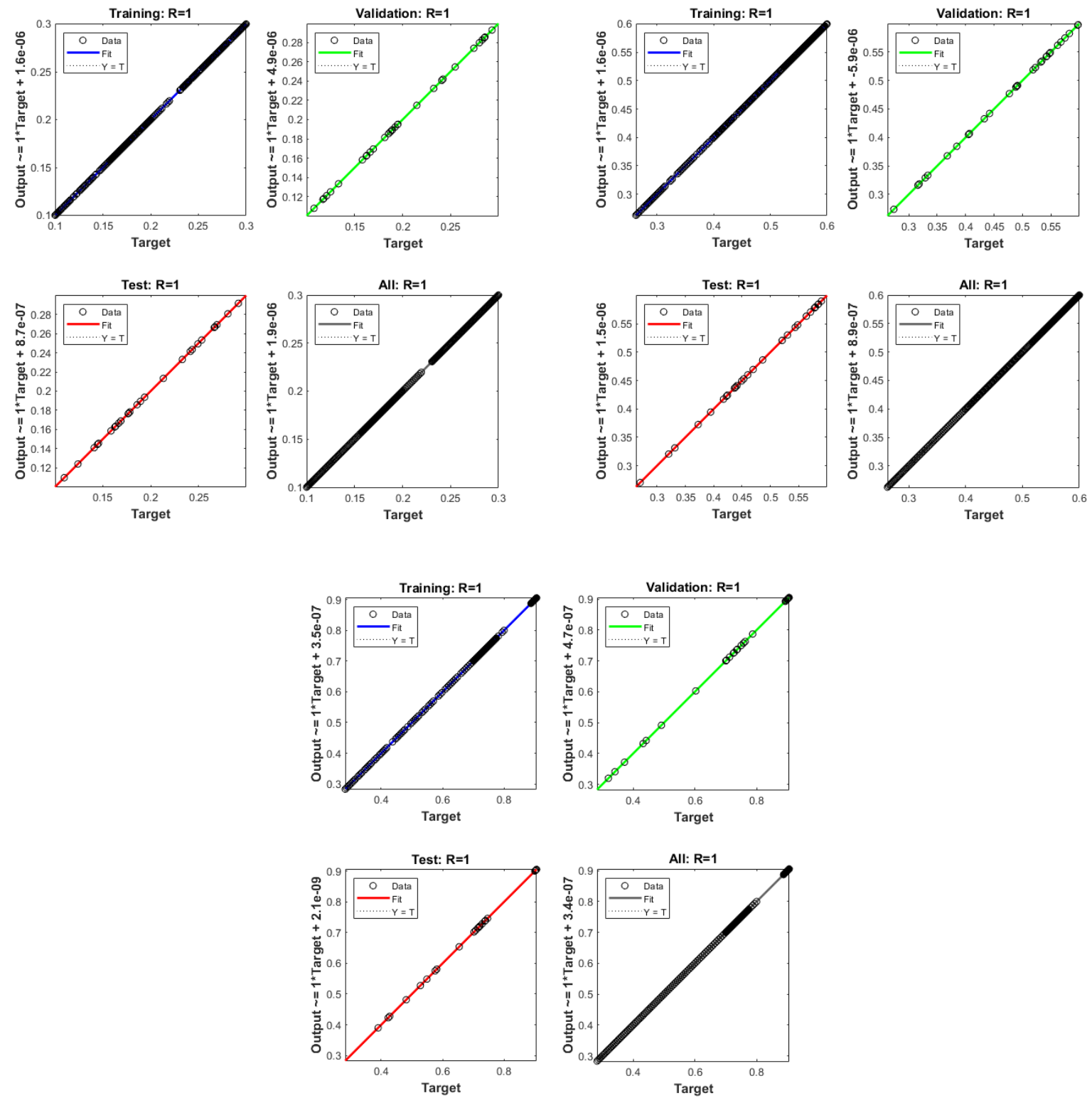

Figure 10: Regression values of the food web system (2) with regards to the designed LMB neural networks in each case.

\section{Research involving human participants and/or animals}

This article does not contain any studies performed on human participants or animals by any of the authors.

\section{Competing interests}

The authors declare that they have no competing interests.

\section{Authors' contributions}

ZS contributed in the conceptualization, formal analysis, methodology, writing, editing and approving the manuscript. NSR involved in formal analysis, methodology and writing the original draft. KK supervised the work and critically revised the manuscript. All authors read and approved the final manuscript. 


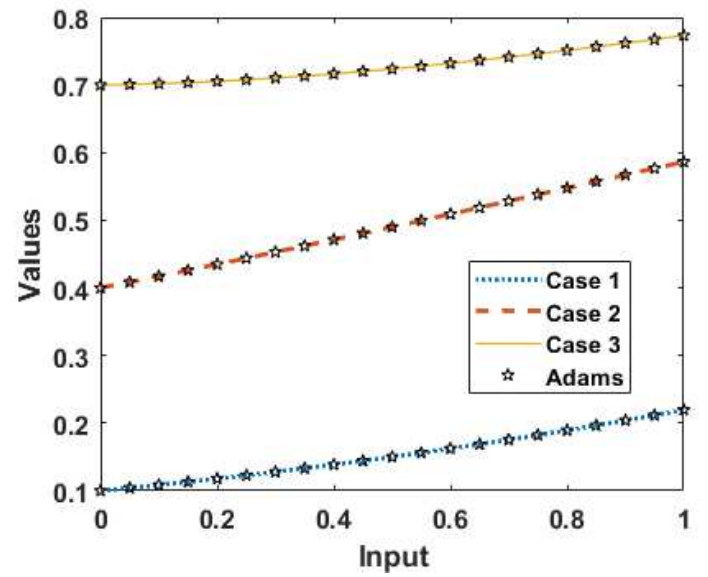

(a) Results of first species $(x(t))$ for all cases of the system (2).

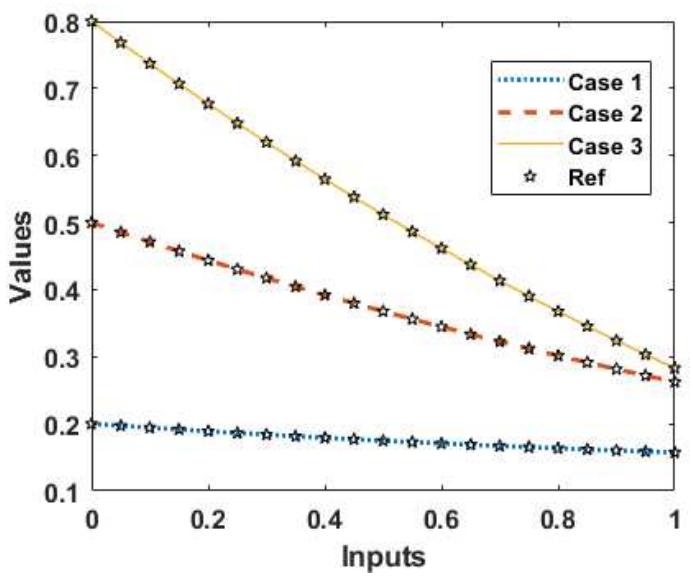

(b) Results of second species $(y(t))$ for all cases of thesystem (2).

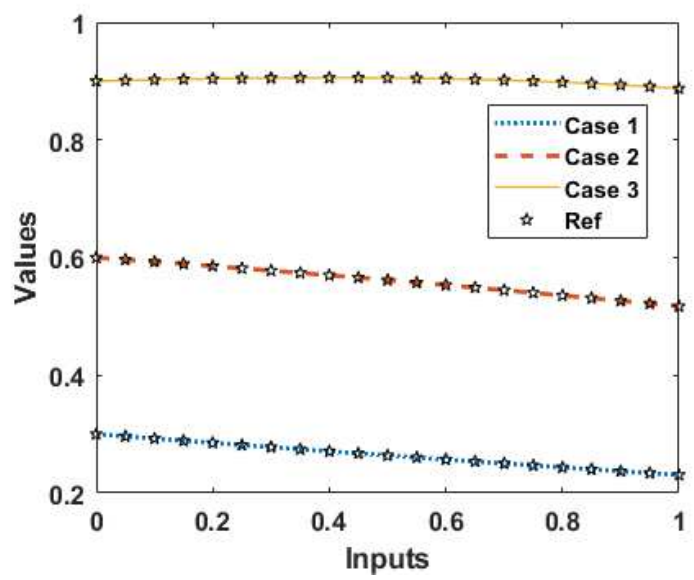

(c) Results of third species $(z(t))$ for all cases of the system (2).

Figure 11: Results comparison in all cases of three species through LMB neural network.

\section{Acknowledgements}

The authors do thankful to the editor for giving an opportunity to submit our research work in this esteemed journal.

\section{References}

[1] G. Seo, D.L. DeAngelis. 2011. A predator-prey model with a Holling type I functional response including a predator mutual interference. J. Nonlinear Sci., 21, pp. 811-833.

[2] G. Peng, Y. Jiang. 2009. Bifurcations of a Holling-type II predator-prey system with constant rate harvesting. Int. J. Bifurc. Chaos, 19, pp. 2499-2514.

[3] S. Yu. 2012. Global asymptotic stability of a predator-prey model with modified Leslie-Gower and Holling-type II schemes. Discret. Dyn. Nat. Soc., 2012, 8 pages.

[4] Y. Huang, F. Chen, L. Zhong. 2006. Stability analysis of a prey-predator model with Holling type-III response function incorporating a prey refuge. Appl. Math. Comput., 182, pp. 672-683.

[5] R.K. Naji, R.N. Shalan. 2013. The dynamics of Holling type IV prey-predator model with intra-specific competitions. Iraqi J. Sci., 54, pp. 386-396. 


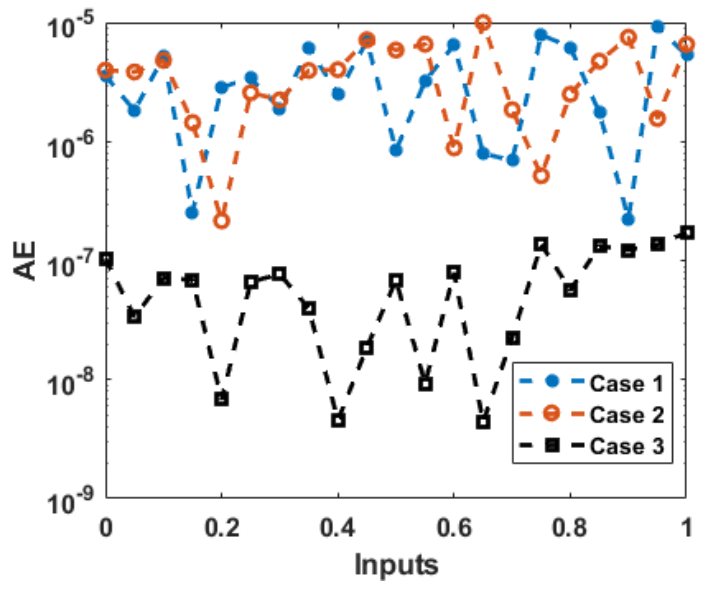

(a) AE of $x(t)$ for all cases of system (2).

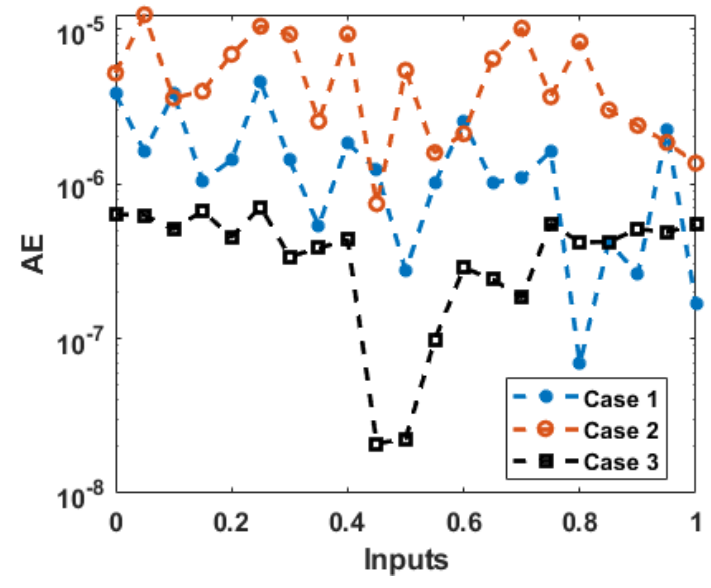

(b) AE of $y(t)$ for all cases of system (2).

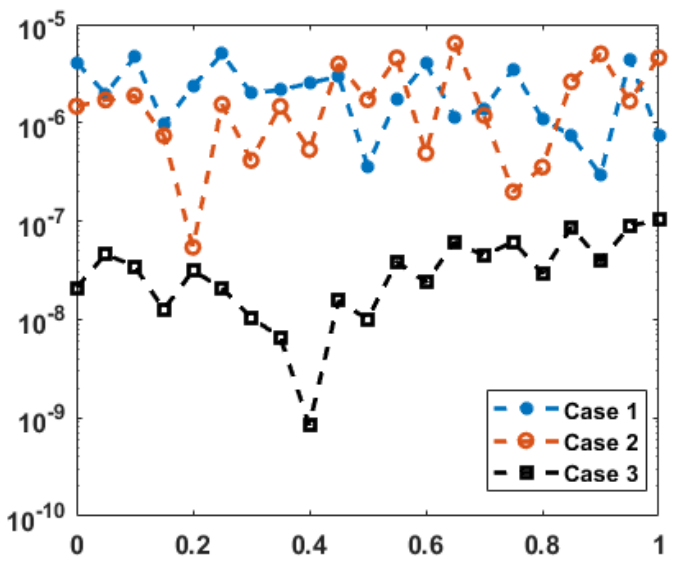

(c) $\mathrm{AE}$ of $z(t)$ for all cases of system (2).

Figure 12: AE based on the obtained results and Adam results via LMB neural network for each case of the system (2).

[6] F. Chen, Z. Ma, H. Zhang. 2012. Global asymptotical stability of the positive equilibrium of the Lotka-Volterra prey-predator model incorporating a constant number of prey refuges. Nonlinear Anal. Real World Appl., 13, pp. 2790-2793.

[7] M. Aziz-Alaoui, M.D. Okiye. 2003. Boundedness and global stability for a predatorprey model with modified Leslie-Gower and Holling type-II schemes. Appl. Math. Lett., 16, pp. 1069-1075.

[8] S. Yu. 2014. Global stability of a modified Leslie-Gower model with BeddingtonDeAngelis functional response. Adv. Differ. Equ., 2014, pp. 1-14.

[9] D. Xiao, W. Li, M. Han. 2006. Dynamics in a rati-dependent predator-prey model with predator harvesting. J. Math. Anal. Appl., 324, pp. 14-29.

[10] M. Sen, M. Banerjee, A. Morozov. 2012. Bifurcation analysis of a ratio-dependent prey-predator model with the Allee effect. Ecol. Complex., 11, pp. 12-27.

[11] S.B. Hsu, T.W. Hwang, Y. Kuang. 2001. Global analysis of the Michaelis-Mententype ratio-dependent predator-prey system. J. Math. Biol., 42, pp. 489-506.

[12] R.S. Cantrell, C. Cosner. 2001. On the dynamics of predator-prey models with the Beddington-DeAngelis functional response. J. Math. Anal. Appl., 257, pp. 206-222.

[13] F. Chen, L. Chen, X. Xie. 2009. On a Leslie-Gower predator-prey model incorporating a prey refuge. Nonlinear Anal. Real World Appl., 10, pp. 2905-2908. 
[14] L. Chen, F. Chen, L. Chen. 2010. Qualitative analysis of a predator-prey model with Holling type II functional response incorporating a constant prey refuge. Nonlinear Anal. Real World Appl., 11, pp. 246-252.

[15] T.K. Kar. 2005. Stability analysis of a prey-predator model incorporating a prey refuge. Commun. Nonlinear Sci. Numer. Simul., 10, pp. 681-691.

[16] J. Pastor. 2011. Mathematical Ecology of Populations and Ecosystems. John Wiley \& Sons.

[17] J. Chattopadhyay. 1996. Effect of toxic substances on a two-species competitive system. Ecol. Model., 84, pp. 287-289.

[18] O. Flaaten. 1991. Bioeconomics of sustainable harvest of competing species. J. Environ. Econ. Manag., 20, pp. 163-180.

[19] T.K. Kar, K. Chaudhuri. 2003. On non-selective harvesting of two competing fish species in the presence of toxicity. Ecol. Model., 161, pp. 125-137.

[20] N. Seshagiri Rao, K.V.L.N. Acharyulu, K. Kalyani. 2015. Numerical study on ecological commensalism between two species with harvested Commensal. ARPN Journal of Engineering and Applied Sciences, 10(4), pp. 1548-1551.

[21] N. Seshagiri Rao, K.V.L.N. Acharyulu, K. Kalyani. 2015. Phase Plane Analysis of A Host-Commensal Ecological Model. International Journal of Advanced Science and Technology, 78, pp. 59-66.

[22] N. Seshagiri Rao, K.V.L.N. Acharyulu, K. Kalyani. 2015. A Host -Mortal Commensal Species Pair With Limited Resources - A Numerical Study. International Journal of Bio-Science and Bio-Technology, 7(1), pp. 133-140.

[23] M. Mamat, W.M. Sanjaya, Z. Salleh, M. Ahmad. 2011. Numerical simulation dynamical model of three-species food chain with Lotka-Volterra linear functional response. J. Sustain. Sci. Manag., 6, pp. 44-50.

[24] E. Chauvet, J.E. Paullet, J.P. Previte, Z. Walls. 2002. A Lotka-Volterra three-species food chain. Math. Mag., 75, pp. 243-255.

[25] A.B. Peet, P.A. Deutsch, E. Peacock-López. 2005. Complex dynamics in a three-level trophic system with intraspecies interaction. J. Theor. Biol., 232, pp. 491-503.

[26] D. Mukherjee. 2014. The effect of prey refuges on a three species food chain model. Differ. Equ. Dyn. Syst., 22, pp. 413-426.

[27] M. Sunaryo, Z. Salleh, M. Mamat. 2013. Mathematical model of three species food chain with Holling type-III functional response. Int. J. Pure Appl. Math., 89, pp. 647-657.

[28] S. Sarwardi, P.K. Mandal, S. Ray. 2012. Analysis of a competitive prey-predator system with a prey refuge. Biosystems, 110, pp. 133-148.

[29] R.K. Upadhyay, S.N. Raw. 2011. Complex dynamics of a three species food-chain model with Holling type IV functional response. Nonlinear Anal. Model. Control, 16, pp. 353-374.

[30] M.A. Aziz-Alaoui. 2002. Study of a Leslie-Gower-type tritrophic population. Chaos Solitons Fractals, 14, pp. 1275-1293.

[31] A. Priyadarshi, S. Gakkhar. 2013. Dynamics of Leslie-Gower type generalist predator in a tri-trophic food web system. Commun. Nonlinear Sci. Numer. Simul., 18, pp. $3202-3218$.

[32] S. Gakkhar, R.K. Naji. 2005. Order and chaos in a food web consisting of a predator and two independent preys. Commun. Nonlinear Sci. Numer. Simul., 10, pp. 105-120.

[33] J. Alebraheem, Y. Abu-Hasan. 2012. Persistence of predators in a two predators-one prey model with non-periodic solution. Appl. Math. Sci., 6, pp. 943-956. 
[34] S. Gakkhar, B. Singh, R.K. Naji. 2007. Dynamical behavior of two predators competing over a single prey. Biosystems, 90, pp. 808-817.

[35] N. Seshagiri Rao, K. Kalyani. 2020. Ecological models on multi Species Interaction within unlimited resources. Int. J. Appl. Comput. Math., 6:95. https://doi.org/10.1007/s40819-020-00847-w

[36] B. Goh. 1979. Stability in models of mutualism. Am. Nat., 113, pp. 261-275.

[37] M. Dhakne, A. Munde. 2012. Stability analysis of mutualistic interactions among three species with limited resources for first species and unlimited resources for second and third species. Differ. Equ. Dyn. Syst., 20, pp. 405-414.

[38] N.P. Kumar, N.C. Pattabhiramacharyulu. 2010. A three species ecosystem consisting of a prey, predator and a host commensal to the prey. Int. J. Open Probl. Comput. Math., 3, pp. 92-113.

[39] N. Seshagiri Rao, N.Ch. Pattabhi Ramachryulu. 2010. Stability of a Syn-Ecosystem consisting of a Prey-Predator and Host Commensal to the Prey (with Mortality Rate for the Prey). International Journal of e Mathematics and Engineering, 1, pp. 411431.

[40] N. Seshagiri Rao, N.Ch. Pattabhi Ramachryulu. 2011. Stability of a Syn- Ecosystem consisting of a Prey-Predator and Host Commensal to the Prey (Mortality Rate for both prey and predator). Acta Ciencia Indica, XXXVII (3), pp. 509-523.

[41] S. Gakkhar, A. Priyadarshi, S. Banerjee. 2012. Role of protection in a tri-trophic food chain dynamics. J. Biol. Syst., 20, pp. 155-175.

[42] Sunita Gakkhar, Komal Gupta. 2016. A three species dynamical system involving prey-predation, competition and commensalism. Applied Mathematics and Computation, 273 , pp. 54-67.

[43] Sabir, Z., et al., 2020. Heuristic computing technique for numerical solutions of nonlinear fourth order Emden-Fowler equation. Math. Comput. Simul., 178, pp. 534-548.

[44] Sabir, Z., et al., 2021. Integrated intelligent computing paradigm for nonlinear multisingular third-order Emden-Fowler equation. Neural Comput. Appl., 33, pp. 34173436 .

[45] Sabir, Z., et al., 2021. Design of neuro-swarming-based heuristics to solve the thirdorder nonlinear multi-singular Emden-Fowler equation. Eur. Phys. J. Plus, 135, pp. $1-17$.

[46] Sabir, Z., et al., 2021. Neuro-evolution computing for nonlinear multi-singular system of third order Emden-Fowler equation. Math. Comput. Simul., 185, pp. 799-812.

[47] M. Umar, Z. Sabir, M.A.Z. Raja. 2019. Intelligent computing for numerical treatment of nonlinear prey-predator models. Appl. Soft Comput., 80, pp. 506-524.

[48] M. Umar, Z. Sabir, F. Amin, J.L.G. Guirao, M.A.Z. Raja. 2020. Stochastic numerical technique for solving HIV infection model of CD4+ T cells. Eur. Phys. J. Plus, 135, 403.

[49] M. Umar, M.A.Z. Raja, Z. Sabir, A.S. Alwabli, M. Shoaib. 2020. A stochastic computational intelligent solver for numerical treatment of mosquito dispersal model in a heterogeneous environment. Eur. Phys. J. Plus, 135, pp. 1-23.

[50] M. Umar, F. Amin, H.A. Wahab, D. Baleanu. 2019. Unsupervised constrained neural network modeling of boundary value corneal model for eye surgery. Appl. Soft Comput., 85, 105826.

[51] H. Ilyas, I. Ahmad, M.A.Z. Raja, M.B. Tahir, M. Shoaib. 2019. Intelligent Computing for the Dynamics of Fluidic System of Electrically Conducting $\mathrm{Ag} / \mathrm{Cu}$ Nanoparticles with Mixed Convection for Hydrogen Possessions. Int. J. Hydrogen Energy, 46, pp. $4947-4980$. 
[52] I. Jadoon, A. Ahmed, A. Rehman, M. Shoaib, M.A.Z. Raja. 2020. Integrated metaheuristics finite difference method for the dynamics of nonlinear unipolar electrohydrodynamic pump flow model. Appl. Soft Comput., 97, 106791.

[53] A. Mehmood, A. Zameer, S.H. Ling, A.U. Rehman, M.A.Z. Raja. 2020. Integrated Computational Intelligent Paradigm for Nonlinear Electric Circuit Models Using Neural Networks, Genetic Algorithms and Sequential Quadratic Programming. Neural Comput. Appl., 32, pp. 10337-10357.

[54] Sabir, Z., et al., 2020. Design of stochastic numerical solver for the solution of singular three-point second-order boundary value problems. Neural Comput. Appl., 33, pp. $2427-2443$.

[55] Sabir, Z., et al., 2020. A Neuro-Swarming Intelligence-Based Computing for Second Order Singular Periodic Non-linear Boundary Value Problems, Front. Phys., 8, 224.

[56] A. Ayub, H.A. Wahab, S.Z. Shah, S.L. Shah, A. Darvesh, A. Haider, Z. Sabir. 2021. Interpretation of infinite shear rate viscosity and a nonuniform heat sink/source on a 3D radiative cross nanofluid with buoyancy assisting/opposing flow. Heat Transfer, 50(5), pp. 4192-4232.

[57] A. Ayub, Z. Sabir, G.C. Altamirano, R. Sadat, M.R. Ali. 2021. Characteristics of melting heat transport of blood with time-dependent cross-nanofluid model using Keller-Box and BVP4C method. Engineering with Computers, pp.1-15.

[58] T. Sajid, S. Tanveer, M. Munsab, Z. Sabir. 2021. Impact of oxytactic microorganisms and variable species diffusivity on blood-gold Reiner-Philippoff nanofluid. Applied Nanoscience, 11(1), pp. 321-333.

[59] T. Sajid, S. Tanveer, Z. Sabir, J.L.G. Guirao. 2020. Impact of activation energy and temperature-dependent heat source/sink on maxwell-sutterby fluid. Mathematical Problems in Engineering, 2020.

[60] T. Sajid, Z. Sabir, S. Tanveer, A, Arbi, G.C. Altamirano. 2020. Upshot of radiative rotating Prandtl fluid flow over a slippery surface embedded with variable species diffusivity and multiple convective boundary conditions. Heat Transfer.

[61] A. Elsonbaty, Z. Sabir, R. Ramaswamy, W. ADEL. 2021. Dynamical analysis of a novel discrete fractional SITR model for COVID-19. Fractals, p.2140035.

[62] Sabir, Z.,et al., 2021. A novel design of fractional Meyer wavelet neural networks with application to the nonlinear singular fractional Lane-Emden systems. Alexandria Engineering Journal, 60(2), pp. 2641-2659.

[63] Sabir, Z., et al., 2021. Fractional Mayer Neuro-swarm heuristic solver for multifractional Order doubly singular model based on Lane-Emden equation. Fractals.

[64] Sabir, Z., et al., 2020. Fractional Meyer neuro-evolution-based intelligent computing solver for doubly singular multi-fractional order Lane-Emden system. Computational and Applied Mathematics, 39(4), pp.1-18. 